\title{
Clinical Illusion: A Giant Nuerofibroma of Diaphragm Mimicking Gastric Mass
}

Sanjay Kumar Yadav*, Gautama Chandra, Kumar Gaurav, Arun Kumar Tiwary and Om Prakash

Department of General Surgery, Rajendra Institute of Medical Sciences, Ranchi, Jharkhand, India

\begin{abstract}
Diaphragm is a quite rare anatomical location for a neuro-fibroma. We encountered a female patient who presented with features of a gastric neoplasm. Upon investigations it turned out to be a case of giant neuro-fibroma of left diaphragm. This case highlights the unusual presentation of a diaphragmatic neurogenic tumor and how it can be mistaken as an gastric mass.
\end{abstract}

\section{Introduction}

Only about 150 cases of primary tumors of diaphragm have been reported in literature to date [1,2]. Primary neural tumors of the diaphragm are very rare, representing approximately $10 \%$ of the neoplasms of the diaphragm. Since the first report of Klassen et al. [3], only 13 cases of diaphragmatic neural tumors have been reported up to date [4].

Diaphragmatic tumors still present as a diagnostic dilemma and are difficult to diagnose preoperatively. They are known to mimic other large masses arising from the mediastinum or abdominal cavity in the majority of cases. We report herein a unique case of a rarely occurring giant neuro-fibroma of the diaphragm, which was successfully excised and treated. Review of literature is also presented.

\section{Case Report}

A 40-years old female patient reported with painless swelling in upper half of abdomen for the last one year. The lady also reported associated symptoms like discomfort after eating and repeated vomiting since the last 4-5 months because of swelling. Although she could not quantify it, but she gave history of weight loss. There was no history of haemetemesis or maleana. On per abdominal examination a firm swelling was found extending from epigastrium superiorly, laterally to right and left both hypochondrium and encroaching to right and left upper half of both lumbar regions inferiorly. Palpating fingers could not be insinuated between the lump and the upper border, as well as the costal margin. Laboratory tests showed normocytic normochromic picture with mild anaemia $(\mathrm{Hb} \% 9 \mathrm{gm} / \mathrm{dl})$. All other tests, such as haemogram, kidney function tests and liver function tests, were within the normal reference ranges.

A Contrast Enhanced Computed Tomography (CECT) scan of the abdomen was performed and showed a large heterogenous mass of size $21.2 \times 15.8 \times 18.4 \mathrm{~cm}$ in the upper abdomen. On post contrast study heterogenous enhancement of the mass was seen. Few non-enhancing areas were noted within the mass suggestive of necrosis. The mass was compressing liver, gall bladder, pancreas and bowel loops. No lymphadenopathy was noted. Upper esophago-gastroscopy was done which revealed extraluminal mass compressing the anterior gastric wall.

$\mathrm{X}$-Ray of chest (PA view) revealed left sided mediastinal shifting. Elective laparotomy was done after routine surgical profile through chevron (roof top) incision. A whitish firm mass was found originating from the right infra-diaphragmatic region. Liver was pushed downwards left infero-laterally and was free from the mass, stomach and small intestine were found compressed by the mass and were free from the mass. Complete resection of the mass was done with reconstruction of diaphragm by putting prolene mesh and right sided chest tube drain was given which was removed after two days.

Histopathological examination of the tumor showed benign spindle cell tumor consisiting of short bundles of benign spindle cells with focal areas of myxoid changes and ill-defined storiform patterns. The spindle cells are separated by moderate amount of collagenous stroma and show long, slender, curved and monomorphic nuclei with pointed ends. These findings were consistent with neuro-fibroma which was proved by Immunohistochemistry positivity for S -100 and negativity for CD34, EMA, CD99, smooth muscle antigen, desmin, and P53.

Patient was discharged on $7^{\text {th }}$ post-operative day. She is in follow up after 1 year of surgery and is doing well.

\section{Discussion}

Grancher was the first to discover the primary tumor of the diaphragm during an autopsy study [5]. Wiener and Chou [6], who documented 71 diaphragmatic tumors, reported the larger series for the first time. Giant diaphragmatic neuro-fibromas are very rare tumors. Only two similar report can be found in the literature $[7,8]$. Article reported a giant neuro-fibroma of the diaphragm, but it is published in a non-English journal [7] and second case was identified only during autopsy [8]. The majority of diaphragmatic tumors are benign. They include mostly lipoma, fibroma and exceedingly rare neuro-fibroma and schwannoma.

Patients with diaphragmatic tumors often present between 40 and 60 years old. Men and women are affected equally. Most patients are symptomatic, with chest wall or abdominal pain, cough, and dyspnea as the most encountered symptoms. Our patient had symptoms of gastric mass. A differential diagnosis of lung, mediastinal, pleural, vertebral or upper abdominal masses has to be kept in mind. Secondary involvement of the diaphragm from adjacent structures may also be

*Corresponding author: Sanjay Kumar Yadav, Department of General Surgery, Rajendra Institute of Medical Sciences, Ranchi, Jharkhand, 834009, India, Tel: +918579002240; Fax: +916512544085; E-mail: sky1508@gmail.com

Received: September 30, 2015; Accepted: October 27, 2015; Published: October 30, 2015

Citation: Yadav SK, Chandra G, Gaurav K, Tiwary AK, Prakash O (2015) Clinical Illusion: A Giant Nuerofibroma of Diaphragm Mimicking Gastric Mass. Oncol Cancer Case Rep 1:102

Copyright: $\odot 2015$ Yadav SK, et al. This is an open-access article distributed under the terms of the Creative Commons Attribution License, which permits unrestricted use, distribution, and reproduction in any medium, provided the original author and source are credited. 
Citation: Yadav SK, Chandra G, Gaurav K, Tiwary AK, Prakash O (2015) Clinical Illusion: A Giant Nuerofibroma of Diaphragm Mimicking Gastric Mass. Oncol Cancer Case Rep 1:102.

present. Symptoms are usually produced due to esophageal, stomach or mediastinal compression.

CT scan findings are well described for benign neurogenic tumors and are first line of investigative modalities. Generally these tumors are well encapsulated and homogenous. CECT delineates the mass and rules out other pathology or extension of the tumor into other organs. CT or USG guided biopsy can establish the diagnosis, especially for suspected lipomas; but most authors prefer thoracotomy/laparotomy to establish and correlate the diagnosis of diaphragmatic tumors with the treatment [9].

Histologically, this was a spindle cell neoplasm. Given its location and its gross and microscopic appearance, it was very difficult to distinguish between neuro-fibroma, solitary fibrous tumor, synovial sarcoma, leiomyoma and mesothelioma. As a result of the immunohistochemical work-up, the tumor was found to be a neurofibroma. Immunochemically, the tumor cells were positive for S100 and negative for CD34, EMA, CD99, smooth muscle antigen, desmin, and P53. Among the differential diagnoses, only neuro-fibroma/ neurofibrosarcoma and synovial sarcoma are usually positive for S100. In addition, the negative staining results for EMA and CD99 ruled out synovial sarcoma. Considering the presence of focal atypical cells, areas of hypercellularity, the low mitosis rate and negative staining for P53, this lesion was diagnosed as neuro-fibroma.

Primary diaphragmatic nuero-fibroma have a good prognosis. Treatment options include surgery followed by radiotherapy and, in some cases, chemotherapy to shrink tumor bulk, followed by resection. These tumors are usually resistant to chemotherapy and radiotherapy with surgical resection being the treatment of choice [10].

\section{Learning Points}

Although rare, diaphragmatic neurogenic tumors should also be included in the differential of atypical appearing masses in the upper abdomen. To our knowledge, this is only the third such case reported to date

\section{References}

1. Evans HL (2011) Low-grade fibromyxoid sarcoma: a clinicopathologic study of 33 cases with long-term follow-up. Am J Surg Pathol 35: 1450-1462.

2. Midorikawa Y, Kubota K, Mori M, Koyama H, Aihara N, et al. (1998) Rhabdomyosarcoma of the diaphragm: report of an adult case. Jpn $\mathrm{J}$ Clin Oncol 28: 222-226.

3. Klassen KP, Patton R, Bemen FM (1945) Neurofibroma of the diaphragm. J Thorac Surg 14: 407-13.

4. McClenathan JH, Okada $\mathrm{F}$ (1989) Primary neurilemmoma of the diaphragm. Ann Thorac Surg 48: 126-8.

5. Grancher M (1868) Extraits des proces-verbaux, Tumeur du diaphragm (8thedn), Bull Soc Anat, Paris.

6. Wiener MF, Chou WH (1965) Primary tumors of the diaphragm. Arch Surg 90:143-52.

7. Korolev AA, Golubev OA (2002) A giant neurofibroma of the diaphragm. Arkh Patol 64: 49-50.

8. Guo R, Chen F, Heffner R (2009) A Giant Atypical Neurofibroma in the Right Thoracic Cavity of a 57-Year-Old Man: A Case Report with Review of the Literature. North American Journal of Medicine and Science 2: 135-138.

9. Mandal AK, Lee H, Salem F (1988) Review of primary tumors of the diaphragm Journal of the national medical association 80: 214-216.

10. McHenry CR, Pickleman J, Winters G, Flisak ME (1988) Diaphragmatic neurilemmoma. J Surg Oncol 37: 198-200. 